\title{
A Novel Algorithm for Dynamic Control System of Hysteretic Power of Shock Absorber
}

\author{
Yiqiang Li \\ Department of Mechanical and Electrical Engineering, Luoding Polytechnic, \\ Luoding527200, China
}

Received: August 19, 2020. Revised: February 22, 2021. Accepted: March 14, 2021. Published: March 30, 2021.

\begin{abstract}
Mechanical resonance and high-frequency vibration caused by the external environment will not only affect the performance of vehicles, ships and machinery, but also cause serious harm to human health. In order to pursue higher ride comfort and reduce the adverse effects on the operator when operating the equipment, the shock absorbers are widely used in aircraft, vehicles and other vehicles or precision machinery. The automatic control of the hysteresis power system of the shock absorber is one of the key tasks in the design and application of the shock absorber. With the development and maturity of mechanical automation technology, PLC control technology has been applied in shock absorbers, but PLC control technology is difficult to achieve the requirements of hysteretic control of shock absorbers in the system precision control. Therefore, a novel algorithm for dynamic control system of hysteretic power of shock absorber is presented. The simulation results show that the control system can effectively improve the control accuracy, and the hysteresis output power of the system is closer to the ideal value.
\end{abstract}

Keywords - shock absorber, hysteretic force, dynamic matrix algorithm

\section{INTRODUCTION}

$\mathrm{W}$ ITH the development of industrial industry, manufacturing industry and precision instrument processing industry, high frequency and irregular vibration will wreck the physical construction of vehicles, ships and equipment, and also wreck the safety performance. For large-scale mechanical power structures such as vehicles and ships, the effects of equipment operation, power output, and even external environmental factors such as airflow and seawater pressure are highly prone to resonance, which in turn causes vibrations in vehicle and ship equipment [1-2]. At the same time, the vibration phenomenon is also a major source of system noise and mechanical noise. With the continuous improvement of the importance of vehicle and ship sonar detection technology and wireless information transmission technology, the system resonance should be suppressed, noise interference should be reduced, and the voice and requirements for improving signal acquisition, recognition and transmission accuracy should be continuously improved [3-4].

In 1995, Besinger F H and Cebon D created a mathematical model for simulation of a heavy vehicle shock absorber. The model has seven parameters determined by the simple dynamic measurement of the test damper, and the mathematical model is verified by the actual working condition of the test bench [5]. However, because the model is created from a specific heavy vehicle suspension damper, the model is only applicable to this kind of model. In 1998, Duym S created a physical model with 10 adjustable valve parameters to simulate the influence of temperature on the viscosity of shock absorber oil and then on the nonlinear dynamic characteristics of automobile shock absorber. The model parameters are also obtained through experiments, and the model is an empirical model [6]. In 2000, D.J. Purdy created a nonlinear model of adjustable automobile shock absorber. The model added parameters such as the compressibility of shock absorber oil, oil-gas emulsification and the expansion and contraction of oil cylinder, and carried out simulation research under the condition of changing these parameters on the performance [7]. In 2001, Tallec and mouro proposed that fluid and structure should be regarded as a unique continuous medium to overcome the calculation difficulty of viscous flow at structural interface. Lagrange method was used to deal with data structure and Euler formula, and fluid structure coupling algorithm was derived, which was embedded in software fire (CFD) for shock absorber simulation [8]. In 2005, Fabio P. Martins adopted CFD model. In 2018, Jan Łuczko and Urszula Ferdek improved the model of a double cylinder oil damper used in 4WD vehicles [9-10]. This model changes the traditional passive control to active control, but it has not been applied to the locomotive, which provides the development of 
locomotive and rolling stock damper.

In the paper, a novel algorithm for dynamic control system is used to analyze the hysteretic power of the shock absorber. The hardware structure and control algorithm implementation flow of the system are designed to improve the control effect on hysteretic power.

\section{HARDWARE DeSIGN OF HYSTERESIS POWER CONTROL SYSTEM FOR SHOCK ABSORBER}

The real-time control of the hysteresis power system of the shock absorber combines the vibration element, the elastic element and the damping element, and then places it between the vibration source and the mechanical structure to form a simple passive damping device. The shock absorber device is mainly composed of a high-performance rubber elastic part, an air elastic element module and the like[11]. The passive shock absorber has the advantages of high reliability, simple structure and low cost, but the vibration elimination effect of the passive shock absorber is weaker [12]. However, once the passive shock absorber is designed, its characteristics and parameter values cannot be corrected or changed. As the use time is extended, the shock absorption performance will be rapidly attenuated, and in the control of the hysteretic power system of the passive shock absorber, it is impossible to adjust the hysteresis power of the shock absorber by controlling the mode of the system parameters. In order to actively control the system parameters and hysteretic power of the shock absorber, an inertial component is added on the basis of the passive control system, so that the passive vibration control device is connected in parallel with the main control controller. A disturbance source is added to the shock absorber device to improve the damping effect of the control system. The active damping device has better control effect, better adaptability, and can actively adjust the hysteretic power. Therefore, a novel algorithm in the paper is proposed, and the parameter control of damping parts and the control effect control of elastic element parameters are strengthened. The overall control effect of the system is superior to the traditional PLC control algorithm and can provide more accurate output control values. The hardware part of the hysteretic power is composed of main modules such as dynamic control center, servo motor, power sensor, presignal amplifier and data calculation unit. The hardware structure design of the system is shown in Figure 1.

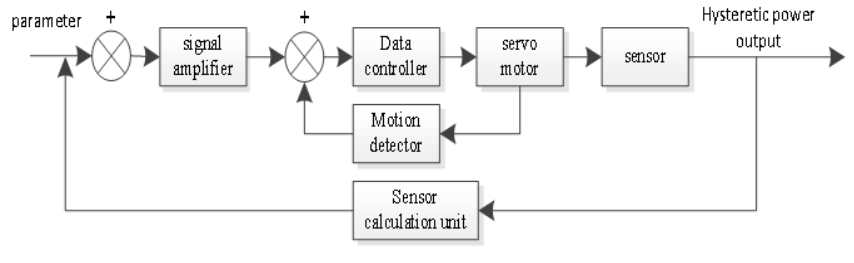

Fig 1. Shock absorber hysteresis power control system hardware structure

The deformation of the elastic component of the shock absorber and the control of the magnitude of the damping value are realized. The front end of the dynamic matrix control center is connected to the signal amplifier connection and the servo motor system, and is indirectly connected with the dynamic displacement sensor and the power control calculation unit to form a multi-way hysteretic power control link. Since the whole hardware system structure adopts the connection mode of the serial interface, the logical operation control result of the dynamic matrix can be cyclically input into the dynamic control center to obtain different hysteretic power output values. Since the whole hardware system structure adopts the connection mode of the serial interface, the logical operation control result of the dynamic matrix can be cyclically input into the dynamic control center to obtain different hysteretic power output values. Before the system commands input to the dynamic control center, the parameters are set and adjusted using electromagnetic signal modulation to reduce the error of the electrical signal. The signal amplifier is used to amplify the electromagnetic signal to reduce the interference of external factors on the system signal. The signal amplifier model related to electromagnetic signal processing is QQ55-FHD-11A, the model of the torque sensor is JN338-V, and the model of the displacement sensor is RDP2536-W.

The logical operation results are based on the input of the dynamic control matrix system, and drives the servo motor module and the torque sensor to work in an electrical signal manner. The MCU controller is built into the servo motor module to adjust the current of the automatic control system, the voltage distribution across the circuit structure, and the rotational speed of the servo motor.

As the power source of the whole shock absorber power control system, the working performance of the servo motor system will determine the working precision of the automatic control system [13-14], which can improve the overall power 
consumption control level and accuracy control level of the servo motor. The servo motor can improve the anti-interference ability and dynamic response speed of the system[15-16]. The dynamic control center has the function of assisting the servo drive device.

This part of the structure is equivalent to a servo drive, and converts three-phase alternating current power into mechanical energy according to the instructions of the control center. The selected servo drive model is SERT32AVC98. This type of drive is designed for the brushless motor structure of the shock absorber power control system, and combines the advantages of servo controllers such as MOSFET and GTR to accurately control the length of the current pulse train and the width of the voltage pulse to achieve precise control for the purpose of the shock absorber hysteresis.

\section{REALIZATION OF Hysteretic POWER System}

The design idea of the control system software takes the time change of hysteretic power as the main line, and uses the loop triggering method to control and trigger each hardware module. First, initialize the entire electronic control system, adjust the input parameters of the system, and then check whether the voltage of the electromagnetic signal control module and the dynamic control center module is stable and the current is normal. If there is a problem, it will be timely feedback to the upper computer of the system for correction. The shock absorber hysteretic power system has two modes: fully automatic and manual control. In the batch detection and control, the automatic mode is used to improve the efficiency, but in the experimental stage, the manual control mode can also be adopted to complete the semi-automatic adjustment of the shock absorber hysteretic power system. The dynamic matrix control method is more flexible. Because the application environment of the shock absorber is very complicated, the dynamic matrix control algorithm can adjust the hysteretic power in a maneuver according to different practical applications. The system master program is shown in Figure 2.

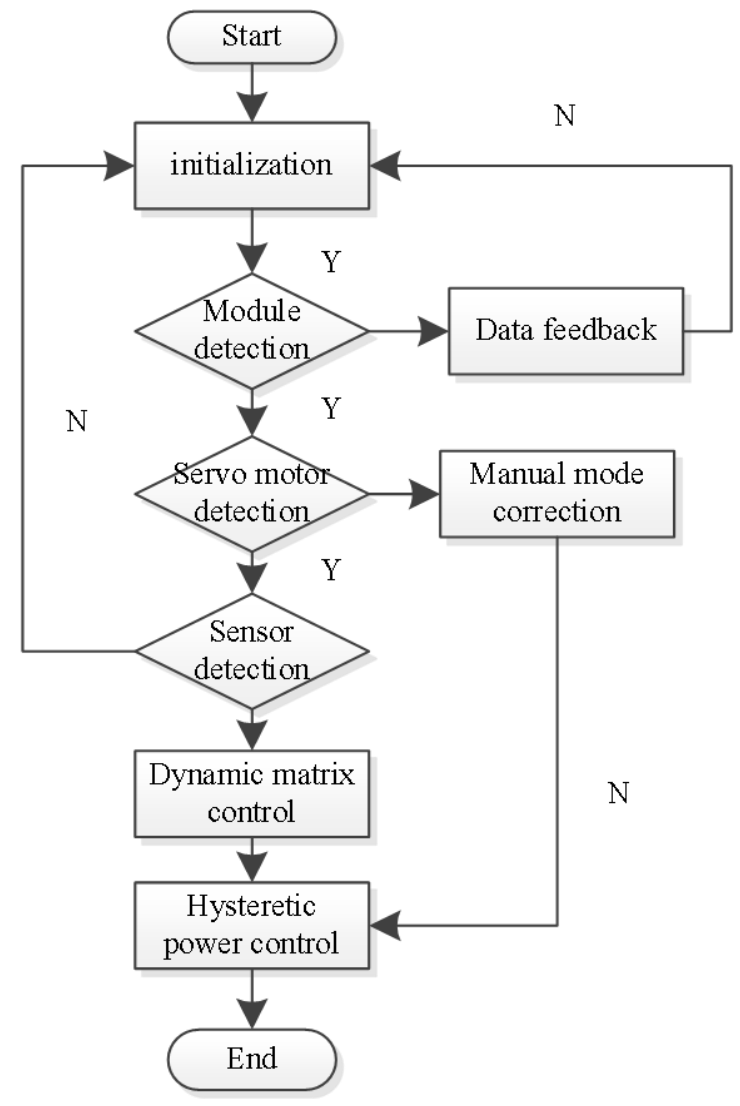

Fig 2. System flow of dynamic matrix control algorithm

After setting the system parameters, determining that the system module and servo motor system, and setting displacement sensor to work properly, the proposed algorithm can realize the optimal control. The proposed algorithm is essentially a pseudo-random control method based on increment to adjust the minimum phase change of the electromagnetic signal, and gradually improve the pure hysteresis performance of the automatic circuit system. The advantage of the novel algorithm is that it overcomes the problem of system parameter delay of the traditional PLC control algorithm, and uses the multi-step prediction method to implement the steps. At the same time, the novel algorithm comprehensively considers the minimum deviation of the system output and the increment of the control variable in the hysteretic control of the shock absorber. The predictive control shows the change behavior of the hysteretic dynamics of the shock absorber in a specific time, so as to accurately obtain the numerical change interval of the control variable. The main function of the predictive control model is to predict the electromagnetic signal impulse response based on the existing hysteretic power change information. The basic process of dynamic matrix prediction for automatic control of shock absorber hysteresis power is shown as Figure 3. 


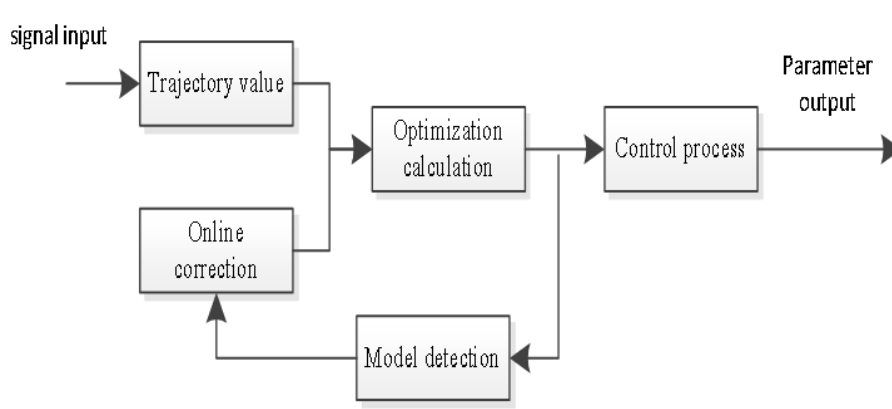

Fig 3. Basic process of dynamic matrix predictive control

After the electromagnetic control signal enters the trajectory numerical system, if there is no abnormal situation, it directly enters the optimization technology stage; if the system recognizes the abnormality, it is corrected online and enters the optimization calculation stage. The optimization calculation uses the calculation method of the dynamic matrix to control the type variables of the elastic element and the damping element to realize the process control of the hysteretic force of the shock absorber, and finally control the change of the hysteretic force in the form of numerical simulation. In the optimization control calculation, the hysteretic force of the shock absorber is taken as the control object, and the step response value of the control system is solved according to the step response characteristics of the hysteretic power. Assuming that $\lambda_{1}, \lambda_{2}, \cdots, \lambda_{\mathrm{t}}$ is a set of step response values, the constant $\mathrm{k}$ simulates the length of the time domain in the system control, where a larger $\mathrm{k}$ indicates that the stability of the step response value is better. The step response value model has nonlinear characteristics and has superposition and proportional variation characteristics, which can obtain the step response control model $\delta_{(i)}$ of the controlled object.

$$
\delta_{(i)}=\lambda_{1} \Delta k(i-1)+\lambda_{2} \Delta k(i-2)+\cdots+\lambda_{t} \Delta k(i-t)
$$

where, $k(i-t)$ is the dynamic matrix control increment of the control system at the $t$ time. At this time, the output value of the shock absorber hysteretic power automatic control prediction model in the future time period can be solved.

$$
\delta_{m}(i+t)=\sum_{t=1}^{m} \lambda_{t} \Delta k(i+t)
$$

The automatic control model of the formula (2) is subjected to dimensionality reduction processing to the complexity of algorithm, and the step response value $\lambda_{t}$ is rewritten into a dynamic matrix of $m \times n$ order.

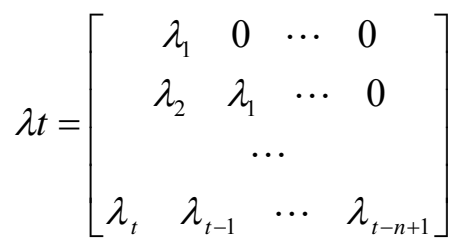

The dynamic matrix control increment of the shock absorber hysteretic power automatic control model can also be expressed by dynamic matrix [17]

$$
\Delta k_{t}=\left[\begin{array}{c}
\Delta k(i) \\
\Delta k(i+1) \\
\cdots \\
\Delta k(t+i-1)
\end{array}\right]
$$

The dynamic matrix expression of the automatic prediction model of the shock absorber can be changed according to the phase change of the pulse signal if a plurality of pulse vectors of the limited amplitude is added, so as to predict the trend of hysteresis hysteretic power output vector in a future interval. However, the prediction result may have multiple related expected outputs. To improve the accuracy of the hysteretic power control, multiple expected outputs are weighted to reduce the deviation between the system output and the theoretical reference value. There is an order of magnitude difference between the predicted power output value deviation and the true output value. Therefore, the penalty function is introduced in the solution of the output value of the binomial expression, which reduces the mutual interference between the reference trajectory and the hysteresis power control output increment, and protects the servo motor controller and the dynamic control center from damage. The hysteretic return control process of the shock absorber also includes a process of rolling optimization. The process of dynamic scrolling optimization is shown in Figure 4.

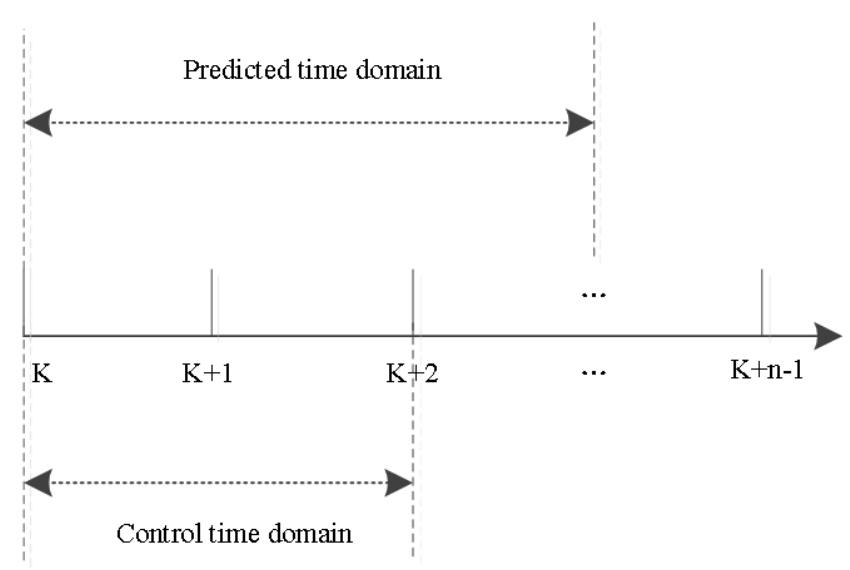

Fig 4. Schematic diagram of rolling optimization of dynamic matrix control algorithm 
The schematic diagram of the rolling control algorithm shows that the expected output result deviates from the actual value, and the rolling optimization algorithm obtains the future $t+i-1$ time control quantity by multiplying the coefficients, and obtains the optimal value of the increment. The incremental control of the dynamic matrix algorithm for objects is only valid within the control time domain, and the risk of failure may occur in all predicted time domains. Therefore, this incremental control must be the continuous control, and the dynamic control of the whole process, that is, the output at the end of the current time is used as the input of the next moment, and the whole process control of the entire motion control process of the shock absorber is realized. The control deviation occurs every time period from YYY to WWW. In the process of adjusting the hysteretic power, the key of dynamic matrix control is to adjust the integral change of the dynamic control increment for the change relationship between the deviation and the increment, and realize a stable full-process dynamic control. The feedback correction link is also one of the key steps in the automatic control of the hysteresis power of the shock absorber. The feedback correction is matched with the feedforward control work, and the compensation control and error warning are provided for the wrong input to ensure the Smooth output of shock absorber hysteretic power [18].

\section{OPTIMIZATION OF HYSTERETIC POWER CONTROL ALGORITHM}

In the paper, two solutions for the displacement-acceleration joint observation and the noise level adaptive evaluation are proposed for the load recognition result with drift phenomenon, and the convergence of the recognition process is improved.

\section{A. The Process of UKF Algorithm}

It is assumed that the state equation and the observation equation of the nonlinear discrete-time system satisfy the following form

$$
\begin{gathered}
\mathrm{x}_{\gamma}=\delta\left(\mathrm{x}_{\gamma-1}, \mathrm{u}_{\gamma-1}, \gamma-1\right)+\mathrm{v}_{\gamma-1} \\
\mathrm{y}_{\gamma}=\varphi\left(\mathrm{x}_{\gamma}, \mathrm{u}_{\gamma}, \gamma\right)+\mathrm{w}_{\gamma}
\end{gathered}
$$

Where $\gamma \in R$ is discrete time, $\mathrm{x} \gamma \in \mathrm{R}^{\mathrm{n}}$ is an $\mathrm{n}$-dimensional random state vector, and $\mathrm{y} \gamma \in \mathrm{R}^{\mathrm{m}}$ is an m-dimensional observation vector.

The nonlinear state equation $\delta($.$) and the nonlinear$ observation equation $\varphi(\cdot)$ are set to be continuous and differentiable at the state quantity $x_{\gamma} . v_{\gamma} \rightarrow n\left(0, q_{\gamma}\right)$ is the process noise, which satisfies the Gaussian distribution, and $w_{\gamma} \rightarrow n\left(0, q_{\gamma}\right)$ is the observed noise, which also satisfies the Gaussian distribution.

The recursive process of the unscented Kalman filter algorithm is as follows

STEP1. Predictive step: $2 \mathrm{n}+1$ sigma points are constructed, the rule is defined as

$$
\left.\bar{x}_{\gamma-1}=\left[m_{\gamma-1}, \cdots, n_{\gamma-1}\right]+\sqrt{n+\lambda} \mid 0, \sqrt{\rho_{\gamma-1}},-\sqrt{\rho_{\gamma-1}}\right]
$$

STEP2. The mean and variance of the next state quantity can be calculated by using UT transform and observation

$$
\bar{x}_{\gamma}=\left[m_{\gamma}^{\prime}, \cdots, m_{\gamma-1}^{\prime}\right]+\sqrt{n+\lambda}\left[0, \sqrt{\rho_{\gamma}^{\prime}},-\sqrt{\rho_{\gamma}^{\prime}}\right]
$$

The Kalman gain is calculated and the state quantity mean and variance are updated

$$
m_{\gamma}=m_{\gamma}^{\prime}+\lambda_{\gamma}\left\lfloor y_{\gamma}-x_{\gamma}\right\rfloor
$$

Where YY represents the observation of step XX. Through the cyclic recursive operation of the above steps, it can be found that the UKF algorithm does not need to linearly transform the nonlinear function, and avoids the operation of the complex Jacobian matrix [19].

\section{B. Load Identification Method}

Due to the uncertainty of the external load and the timevarying amplitude, it is difficult to characterize the external load using a deterministic model in the state space. Considering that the load time history is a stochastic process, the load time history can be decomposed into a linear combination of a series of coefficients and a polynomial orthogonal basis product by orthogonal decomposition, and these coefficients are independent of each other. This decomposition method can effectively quantify the stochastic process and facilitate the establishment of mathematical models for mathematical analysis.

In the process of decomposing the load time history, it is found that the stochastic process decomposition method based on the standard orthogonal basis works well, and can also avoid solving the complex Fredholm integral equation. Chebyshev's orthogonal multiple decomposition is as follows.

$$
T_{m}^{i}=\left\{\begin{array}{c}
\frac{1}{\sqrt{\pi}}, m=0 \\
\sqrt{\frac{2}{\pi}\left(\frac{2 t}{L_{i n p}}-1\right), m=1} \\
2\left(\frac{2 t}{L_{i n p}}-1\right) T_{m}^{i}(t)-T_{m-1}^{i}, m=2,3, \cdots, n
\end{array}\right.
$$


Where, $L_{i n p}$ is the input length and $\mathrm{n}$ is the decomposition order. Based on the standard polynomial decomposition, the structural motion equation is as follows.

$$
m x^{\prime}(t)+c x^{\prime}(t)+n x^{\prime}(t)=L \sum_{\gamma=1}^{f} \sum_{\rho=1}^{\lambda} w_{m}^{n} t_{m}^{n}(t)
$$

That is, the state space equation of the UKF algorithm based on the load orthogonal decomposition can be written as follows.

$$
\theta_{i}=\left[\begin{array}{c}
x^{\prime} \\
x^{\prime \prime} \\
\bar{y}
\end{array}\right]=\left\lfloor m-1\left[L \sum_{\gamma=1}^{n} \sum_{\gamma \in 1}^{m} w_{m}^{n} t_{m}^{n}-\left(c x_{t}\right)-\left(\lambda_{i-1}\right)\right]\right\rfloor
$$

The role of the state variables $\gamma$ and $\lambda$ in the equation is to identify the stiffness and damping of the structural system. If no identification is required, it can be removed, that is, only the orthogonal expansion factor $\mathrm{w}$ is left.

Since the indirect load identification method needs to identify many parameters, and only the orthogonal expansion coefficients are identified, the product of the multi-step coefficient and the orthogonal basis is needed to obtain the load to be identified. In addition to the selection of the number of items is also a problem, it is difficult to unify the conclusion. Therefore, in order to reduce the number of parameters to be identified and to simplify the operation process, the external load to be identified can be directly identified as a state variable, that is, a direct load identification method. A linear discrete system is set as follows.

$$
\mathbf{x}_{\gamma+1}=\mathrm{Ax}_{\gamma}+\mathrm{Bp}_{\gamma}+\mathrm{w}_{\gamma}
$$

Where $\mathrm{A}$ and $\mathrm{B}$ are system matrices, $\mathrm{w}_{\gamma}\left(\mathrm{w}_{\gamma} \in \mathrm{R}^{\mathrm{ns}}, 0 \leq \gamma \leq \infty\right)$ is random process noise, and ns is the number of state quantities. To supplement equation (13), a load vector $\rho \in R^{n p}$ is set at time $\gamma$ and $\gamma+1$.

$$
\rho_{\gamma+1}=\rho_{\gamma}+\mu_{\gamma}
$$

Where $\mu_{\gamma}\left(\mu_{\gamma} \in R^{n p}, 0 \leq \gamma \leq \infty\right)$ is a random process component.

By reasonably selecting the covariance matrix of the process noise $\mu_{\gamma}$, the load time history can be estimated using equation (14). The state quantity is redefined as follows.

$$
x_{\gamma}^{\alpha}=\left\lfloor\begin{array}{l}
x_{\gamma} \\
\rho_{\gamma}
\end{array}\right\rfloor
$$

The extended state equation is as follows.

$$
x_{\gamma+1}^{\alpha}=A_{\alpha} x_{\gamma}^{\alpha}+\delta_{\gamma}
$$

The matrix $A_{\alpha}$ is defined as

$$
A_{\alpha}=\left\lfloor\begin{array}{cc}
A & B \\
0 & t
\end{array}\right\rfloor
$$

The noise vector $\ell \gamma \in R^{n_{2}+n_{\rho}}$ depends on the model error $w_{\gamma} \in R^{n}$ and the random increment of the load.

$$
\ell_{\gamma}=\left\lfloor\begin{array}{l}
w_{\gamma} \\
\mu_{\gamma}
\end{array}\right\rfloor
$$

The matrix $C_{\alpha} \in R^{n d\left(n_{3}+n_{\rho}\right)}$ is composed of an output influence matrix $\mathrm{C}$ and a direct conversion matrix $\mathrm{Z}$.

$$
C_{\alpha}=\left[\begin{array}{ll}
C & J
\end{array}\right]
$$

The state equations and observation equation expressions for expanding state quantities are as follows, respectively.

$$
x_{\gamma+1}^{\alpha}=A_{\alpha} x_{\gamma}^{\alpha}+\ell_{\gamma}, \quad d_{\gamma}=C_{\alpha} x_{\gamma}^{\alpha}+v_{\gamma}
$$

where the system matrices $\alpha, \beta, C$ and $\mathrm{J}$ for identification can be obtained according to different methods.

\section{SYSTEM FUNCTION AND PERFORMANCE TEST}

\section{A. Test Platform Construction}

The simulation experiment platform is built to verify the system function and test performance of the system. The platform system mainly includes $10 \mathrm{kV}$ high voltage switchgear, high performance sensor, oscilloscope, transformer with isolation performance, and special tooling fixture. The simulation test is carried out based on the dynamic domain fuzzy control device to verify the steady state performance and control error of the automatic control system.

\section{B. Functional Test of Automatic Control System}

In order to verify the basic control function and circuit overload protection function of the shock absorber hysteresis power control system, and avoid system failure in batch experiments. The functionality of the automation control system needs to be verified, and the experimental data is obtained from the perspectives of local control and remote control, as shown in Table 1. 
Table 1. Basic function test of shock absorber automatic control system

\begin{tabular}{ccccc}
\hline \multicolumn{2}{c}{ Control method } & $\begin{array}{c}\text { Number of } \\
\text { experiments }\end{array}$ & $\begin{array}{c}\text { Number of } \\
\text { successes }\end{array}$ & $\begin{array}{c}\text { Number of } \\
\text { failures }\end{array}$ \\
\hline remote & stretching & 4783 & 4783 & 0 \\
control & $\begin{array}{c}\text { compress } \\
\text { ion }\end{array}$ & 4920 & 4920 & 0 \\
local & stretching & 4775 & 4775 & 0 \\
control & $\begin{array}{c}\text { compress } \\
\text { ion }\end{array}$ & 4920 & 4920 & 0 \\
\hline
\end{tabular}

The test data shows that the automatic control system stretching and compression basic actions proposed in this paper can be successfully completed, whether it is remote control or local control, which indicates that the mechanical function of the system is good. Next, the current overload protection performance of the automatic control system is verified. According to the relationship between current and temperature when the DC motor is overloaded, the threshold setting range of the current overload protection is determined. When the control system circuit is overloaded, the dynamic matrix-based shock absorber hysteretic power system will actively cut off the current to protect the safety of the control system circuit structure. The protective characteristic curve of the servo motor of the control system changes as shown in Figure 5.

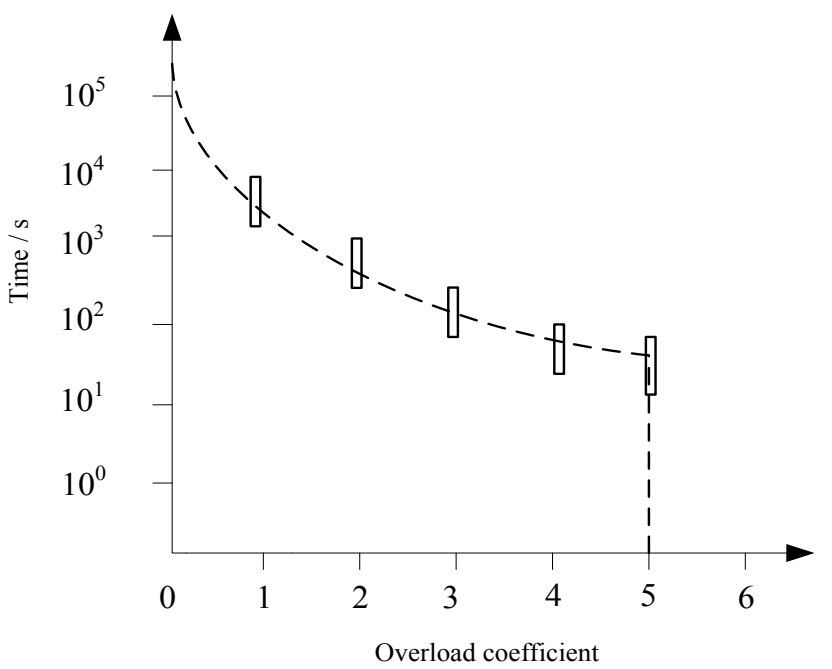

Fig 5. Shock absorber automatic control system overload protection characteristic curve

Since the automatic control system of the shock absorber has overload protection performance, we can draw the conclusion that the current overload multiple is not more than 5 times to ensure the stable operation of the servo motor of the automatic control system according to a large number of experimental data and practical experience. The system function test results show that the dynamic matrix-based shock absorber hysteretic power system has good system function and circuit protection function, which can ensure the stable operation of the experimental system.

\section{System Performance Test Comparison}

The stability of the system is compared and tested. Under different steady-state conditions, the dynamic matrix control system in the paper compares with the traditional PLC control system on the error control level. The selected system steadystate value and error fluctuation amplitude interval are shown in Table 2.

Table 2. Steady-state value setting for system performance test

\begin{tabular}{ccc}
\hline No & steady-state value & $\begin{array}{c}\text { error fluctuation } \\
\text { amplitude interval }\end{array}$ \\
\hline 1 & 0 & {$[-1,1]$} \\
2 & 10 & {$[8,12]$} \\
3 & -10 & {$[-17,-3]$} \\
\hline
\end{tabular}

Compared with the three steady-state values, the change in control error between the traditional control system based on PLC and the dynamic matrix control method proposed in the paper under the simulation time of 900s. Firstly, based on the control error of the three steady-state values of the PLC control system is as shown in Figure 6, where a, b, and c represent the error levels under the steady state values of 0,10 , and -10 , respectively.

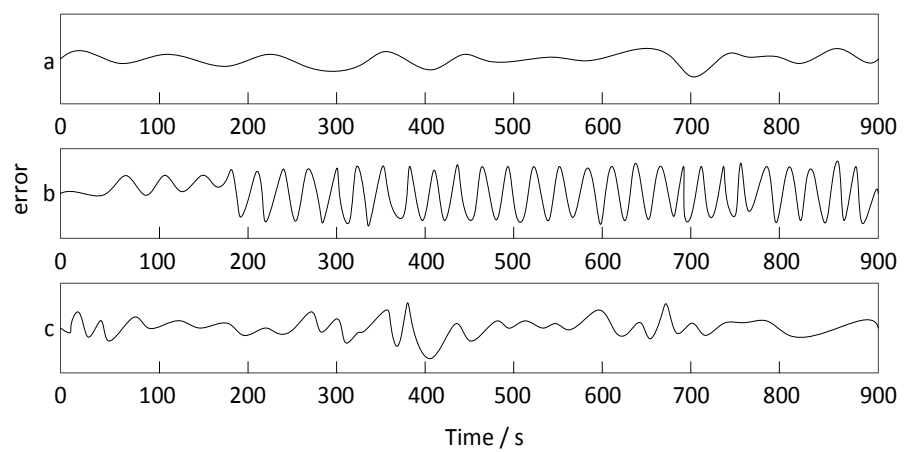

Fig 6. Variations in control error at steady state values 
Different problem values are selected under the traditional PLC control method, and different error outputs are presented under the simulation time of 120 s. Under the condition that the steady-state value is zero, the waveform output of the error is relatively stable, but the control error value is generally high, exceeding \pm 0.1 . When the steady-state value is 10 , the sudden increase of the error occurs in the period of $20 \sim 30 \mathrm{~s}, 60 \sim 70 \mathrm{~s}$, $100 \sim 110$ s, it indicates that fluctuations in steady state values can adversely affect changes in control error. When the steadystate value is -10 , the overall trend of the control error also shows a periodic change, but the overall error mean also exceeds \pm 0.1 .

The error output of the system approaches zero when the steady state value is zero. Under the condition that the steady state value of the system is 10 , the control error output in the 120 s simulation time is less than \pm 0.02 . Under the condition that the steady state value is -10 , the error output of the automatic control system is improved, but it can still be controlled within \pm 0.05 . The specific error output waveform changes are shown in Figure 7.

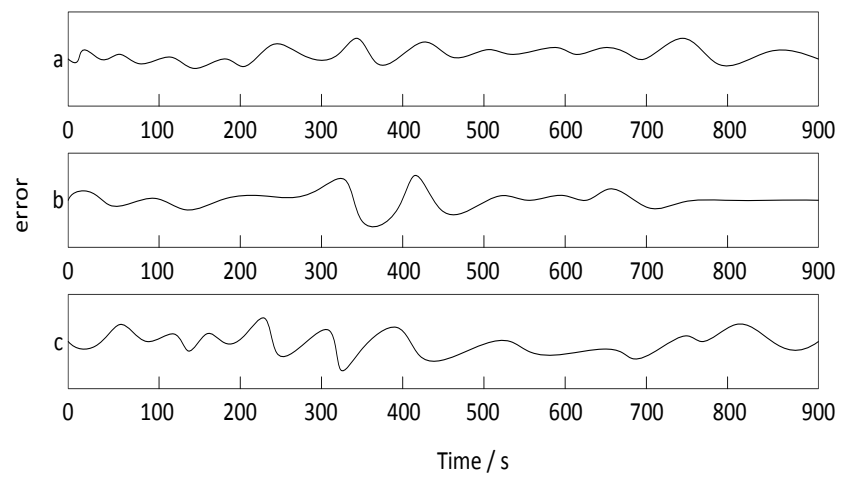

Fig 7. Change of control error under steady-state value

From the experimental analysis results, the dynamic control system of the shock absorber hysteretic power proposed in the paper is functionally stable, and the system is superior to the traditional PLC control method in terms of error control performance.

\section{CONCLUSION}

The existing automatic control system of hysteretic power of vibration isolator based on PLC has the disadvantage of high control error. Therefore, an automatic control system based on dynamic matrix control algorithm is designed. The hardware part of the system consists of dynamic control center, servo motor, power sensor, pre signal amplifier, data calculation unit and other key modules, In order to improve the control performance of the system, STM3254A MCU controller is built in the servo motor module to ensure the stable output of electromagnetic control signal. According to the principle of dynamic matrix control algorithm, the main control program of automatic control system is designed, and the basic process of dynamic predictive matrix control is given. The dynamic rolling predictive mode is used to reduce the error in the control time domain, The analysis data show that the proposed automatic control system can meet the requirements of batch test and remote control in terms of functionality, and the system error under three steady-state values is significantly lower than that of the traditional vibration isolator control system, and the highest error output is between \pm 0.05 .

As a new type of semi-active vibration isolation component, the accurate dynamic model of magnetorheological elastomer (MRE) vibration isolator is the key to achieve good damping performance. The dynamic model of magnetorheological elastomer is highly nonlinear and time-delay. At present, most of the MRF damper used parametric model, according to the experimental data for parameter identification, that is, under a certain current, a certain excitation, if the established parameter model can be better close to the experimental data, it shows that the parameter model can better characterize the mechanical properties of MRF material.

\section{REFERENCES}

[1] Kim W S, Ahn D J, Lee J K. A Study on the Seismic Isolation Systems of Bridges with Lead Rubber Bearings. Open Journal of Civil Engineering, 2014, 04(4):361-372.

[2] Khiabani E D, Ghaffarzadeh H, Shiri B, et al. Spline collocation methods for seismic analysis of multiple degree of freedom systems with viscoelastic dampers using fractional models. Journal of Vibration and Control, 2020:107754631989857.

[3] Weiling, Chen, Ke, et al. Statistical and Structural Information Backed Full-Reference Quality Measure of Compressed Sonar Images. IEEE Transactions on Circuits and Systems for Video Technology, 2019, 30(2):334-348.

[4] Dhaya R, Kanthavel R . A Wireless Collision Detection on Transmission Poles through IoT Technology. Journal of Trends in Computer Science and Smart Technology, 2020, 2(3):165-172.

[5] Besinger F H , Cebon D , Cole D J . Damper Models for Heavy Vehicle Ride Dynamics. Vehicle System Dynamics, 1995, 24(1), p.35-64.

[6] Duym S , Reybrouck K . Physical Characterization of Nonlinear Shock Absorber Dynamics. European journal of mechanical engineering, 1998, 43(4), p.181-188.

[7] Purdy, D J. Theoretical and experimental investigation into an adjustable automotive damper. Proceedings of the Institution of Mechanical Engineers Part D Journal of Automobile Engineering, 2000, 214(3), p.265-283.

[8] P, Le, Tallec, et al. Fluid structure interaction with large structural displacements. Computer Methods in Applied Mechanics and Engineering, 2001, 190(24-25), p.3039-3067.

[9] Luczko Jan, Urszula F. Non-linear analysis of a quarter-car model with stroke-dependent twin-tube shock absorber. Mechanical Systems \& Signal Processing, 2019, 115, p.450-468.

[10] Wang Z, Ding L, Pei T, et al. Large signal operation of small band-gap carbon nanotube-based ambipolar transistor: a high-performance frequency doubler. Nano Letters, 2010, 10(9), p.3648-3655.

[11] Kim Y, Lee J, Park J. A structural optimization of laminated composite curved structure for an elastic shock absorber of UAV based on a novel theory. Mechanics of Advanced Materials and Structures, 2020, In Press. 
[12] Shi F. Multi-objective Optimization of Passive Shock Absorber for Landing Gear. American Journal of Mechanical Engineering, 2019, 7(2):79-86

[13] Helian B, Chen Z, Yao B. Precision Motion Control of a ServomotorPump Direct-Drive Electrohydraulic System With a Nonlinear Pump Flow Mapping. IEEE Transactions on Industrial Electronics, 2020, 67(10):8638-8648.

[14] Li X J , Shen X Y . A Data-Driven Attack Detection Approach for DC Servo Motor Systems Based on Mixed Optimization Strategy. IEEE Transactions on Industrial Informatics, 2020, 16(9):5806-5813.

[15] Gadala I M , Li B , Meng Q H , et al. An expert system for motor sizing using mechanical dynamics and thermal characterization. Control and Intelligent Systems, 2020, 48(1):44-56.

[16] Jiang H, Fu H, Han Z, et al. Elimination of Gear Clearance for the Rotary Table of Ultra Heavy Duty Vertical Milling Lathe Based on Dual Servo Motor Driving System. Applied Sciences, 2020, 10(11):4050.

[17] Giwa A, Owolabi J O, Giwa S O . Dynamic Matrix Control of a Reactive Distillation Process for Biodiesel Production. International Journal of Engineering Research in Africa, 2019, 45:132-147.

[18] Marek Kubalcik, Vladimir Bobal, Tomas Barot, Statistical Analysis of Modified Predictive Control of Non-Minimum Phase System, WSEAS Transactions on Applied and Theoretical Mechanics, Volume 14, 2019, pp. 205-211.

[19] Wen-Jer Chang, Che-Lun Su and Cheung-Chieh Ku, Robust Fuzzy Controller Design with Decay Rate for Nonlinear Perturbed Singular Systems, Engineering World, Volume 1, 2019, pp. 4-8.

\section{Creative Commons Attribution License 4.0 (Attribution 4.0 International, CC BY 4.0)}

This article is published under the terms of the Creative Commons Attribution License 4.0

https://creativecommons.org/licenses/by/4.0/deed.en US 